\title{
Fluctuaciones económicas bajo régimen de cambio fijo en una economía pequeña con dos sectores*
}

\section{Economic fluctuations on fixed rate}

in a small economy with two sectors

José U. Mora Mora**

Recibido: 2 de septiembre de 2012

Aprobado: 16 de enero de 2013

\section{Resumen}

Este trabajo presenta un modelo teórico de oferta y demanda agregada que permite explicar la propagación de los efectos de la política económica y de alteraciones en los términos de intercambio sobre las variables macroeconómicas en una economía pequeña con dos sectores productivos bajo un régimen de cambio fijo. Contrario a los resultados tradicionales, una devaluación de la moneda local produce una contracción económica en el corto plazo y aumenta el nivel de precios. De igual manera, el incremento de los

* Proyecto CDCHTA-ULA: E-237-05-09-C. Deseo agradecer al Consejo de Desarrollo Científico, Humanístico, Tecnológico y de las Artes (CDCHTA) de la Universidad de Los Andes por el financiamiento y el apoyo para la conclusión de este proyecto. Cómo citar este artículo: Mora, J. (2013). Fluctuaciones económicas bajo régimen de cambio fijo en una economía pequeña con dos sectores. Revista CIFE, 15 (22), pp. 141-160.

** Profesor Titular. Instituto de Investigaciones Económicas y Sociales. Universidad de Los Andes. Av. Las Américas, Campus Liria, Edif G. 3er Piso. Mérida, Estado Mérida, ZP 5101. Venezuela. E-mail: jumora@ula.ve 
precios del producto de exportación muestra dos efectos simultáneos. Afecta la oferta agregada por el estímulo a la producción del sector, y por otro lado, acrecenta la demanda agregada. Dependiendo de cuál efecto domine sobre la economía, entonces, los precios domésticos pueden aumentar, disminuir o permanecer inalterados.

Palabras clave: ciclos económicos; política fiscal; política monetaria; política cambiaria.

Clasificación JEL: B22, E12, E32, N56

\section{Abstract}

This paper presents a theoretical model of aggregate supply and demand that helps explain the propagation effects of economic policy and the terms of trade on macroeconomic aggregates in a small open economy with two sectors and under a fixed exchange rate regime. Contrary to traditional results, a devaluation does not affect real economic activity but causes inflation. Likewise, an increase in the price of export product causes two simultaneous effects. Increases aggregate supply because by increasing output in the export sector and rises aggregate demand. Depending on which effect dominates, domestic prices might increase, decrease or remain constant.

Keywords: business cycles; fiscal policy; monetary policy; fixed rates.

Classification JEL: B22, E12, E32, N56 


\section{Introducción}

Las causas de las fluctuaciones de la actividad económica real en una economía abierta son diversas. Por un lado, la política económica del lado de la demanda de la economía tiene efectos limitados que dependen del régimen cambiario y del grado de movilidad de capital. Mientras más elevado sea el grado de movilidad de capital, mayores serán los efectos de la política fiscal bajo un régimen de cambio flotante y superiores serán los efectos de la política monetaria en un régimen de cambio fijo (Mundell, 1963; Fleming, 1962). Evidentemente, estos ajustes tienen lugar sólo si existe rigidez de precios y salarios. Por el contrario, cuando se supone flexibilidad de precios y salarios, entonces, la duración de los efectos de la política fiscal y monetaria se reducen en un todo de acuerdo con los postulados de la teoría económica tradicional.

Los efectos de la política fiscal y monetaria tienden a ser temporales y sólo los shocks de oferta tienen efectos permanentes (véase: Blanchard y Watson, 1986; Blanchard y Quah, 1989; Cecchetti y Karras, 1992; Karras, 1993; 1994; Lorenzoni, 2009; Galí, 1999; King, et al., 1991). Aunque sobre esto último todavía existe mucho debate. Aparentemente, conforme con los postulados de Lucas $^{1}$ una política monetaria no anticipada pudiera tener efectos de largo plazo (véase: Cooley y Hansen, 1997). Por el otro lado, las perturbaciones externas afectan la economía a través de la balanza de pagos. En primer lugar, las perturbaciones externas pueden actuar directamente sobre los términos de intercambio, y de esta manera ocasionar fluctuaciones económicas que pudieran ser permanentes. Generalmente, todos estos trabajos tienen como objetivo formalizar una explicación de la evolución y de las fluctuaciones económicas de Estados Unidos u otra economía desarrollada o altamente industrializada después de la segunda guerra mundial y utilizan los paradigmas neoclásicos para explicar tal comportamiento.

Este trabajo se aleja ligeramente de esa dirección. Este texto trata de explicar más el comportamiento de un país que produce y exporta una materia prima muy importante en el contexto mundial, pero cuyos mercados domésticos no están altamente desarrollados o integrados. El documento construye un modelo que pudiera explicar el comportamiento de algunas economías latinoamericanas o africanas. Estas economías se caracterizan por tener una alta dependencia de un producto de exportación (petróleo o algún otro artículo agrícola o mineral importante). En ese sentido, el modelo teórico de oferta y demanda agregada que se presenta permite explicar la propagación de los efectos de la política económica y de alteraciones en los términos de intercambio sobre las variables macroeconómicas en una economía pequeña monoexportadora.

Este trabajo se organiza de la siguiente manera: en la segunda sección se presentan los supuestos y se construye el modelo. En el aparte tercero se determinan y se analizan

1 Missperception theory. 
los impactos y el mecanismo de propagación de los shocks considerando un régimen de cambio fijo. La cuarta discute las implicaciones de política económica, y la quinta parte concluye.

\section{Elementos del modelo}

\subsection{Supuestos generales}

La estructura del modelo se basa en las contribuciones de Mundell (1963) y Fleming (1962) con la excepción de que, como se verá más adelante, los precios no son rígidos. Específicamente, se supone una economía pequeña que mantiene una actividad comercial con el resto del mundo ya que uno de sus dos sectores productivos se dedica a la extracción, producción y exportación de una materia prima (llámese petróleo, cobre o cualquier otro mineral) muy importante para las economías del resto del mundo. Este producto constituye el único producto de exportación. En ese sentido la economía, como se señaló previamente, se supone está dividida en dos sectores productivos: el sector exportador (sector 1) y la sección que comprende el resto de las actividades que producen otros bienes y servicios (sector 2). Se supone, además, que el precio del producto de exportación es determinado por el mercado mundial, $P_{1, t}^{*}$. La producción del sector 2 se vende en el mercado doméstico a los precios $P_{t}$.

Dado que la economía es pequeña y no está integrada completamente con los mercados financieros internacionales, se sospecha que existe movilidad imperfecta de capitales. Este supuesto tiene implicaciones muy importantes para la determinación del saldo de la balanza de pagos.

Se cree que los precios domésticos $P_{t}$ son flexibles. Adicionalmente, que los sindicatos tienen un elevado poder de mercado que conduce a indexar los salarios de los trabajadores en los diferentes sectores de la economía y utilizan para ello el índice de precios al consumidor (IPC). Supóngase que el IPG se calcula de la siguiente manera:

$$
I P C_{t}=\left(E P_{t}^{*}\right)^{v}\left(P_{t}\right)^{1-v}(1)
$$

Donde,

$v, P_{t}^{*}, P_{t}$ y $E$ son, respectivamente, la ponderación de los precios domésticos en la canasta del IPC, los precios externos (en dólares) y los precios domésticos (en moneda local) usados en la construcción de la canasta y la tasa de cambio nominal en moneda local por dólar. Supóngase la tasa de salarios en el sector $i$ se indexa de la siguiente manera:

$$
W_{i, t}={ }_{i} I P C_{t}={ }_{i}\left(E P_{t}^{*}\right)^{v}\left(P_{t}\right)^{1-v}(2)
$$

Donde, 
$0<i_{i}<1$ es la tasa a la que se indexan los salarios. Si ${ }_{i}=0$ los salarios del sector $\mathrm{i}$ son rígidos y si $i=1$, entonces, los salarios del sector i son completamente flexibles y se ajustan por el cambio en el IPC. Tomando logaritmos ${ }^{2}$ de la expresión anterior se obtiene:

$$
w_{i, t}={ }_{i}+v e+v p_{t}^{*}+(1-v) p_{t}(3)
$$

\subsection{Oferta agregada}

Este modelo supone que el producto real se forma de la siguiente manera:

$$
Y_{t}=E X P^{y_{1, t}+y_{2, t}}
$$

Donde,

$y_{p, t}$ y $y_{o, t}$ son los logaritmos del producto real del sector exportador y del resto de las actividades, respectivamente. Tomando logaritmos naturales de la ecuación (4) se obtiene:

$$
y_{t}=y_{1, t}+y_{2, t}(5)
$$

\subsubsection{Producción en el sector exportador}

La producción en el sector exportador se realiza por medio de una tecnología CobbDouglas con rendimientos constantes a escala:

$$
\Upsilon_{1, t}=Z_{1, t} K_{1}^{\theta} L_{1, t}^{1-\theta}(6)
$$

Donde,

$0<\theta<1, K_{1}$ y $L_{1, t}$, corresponden a la cantidad de capital y trabajo empleado en el sector en el momento $t$ y $Z_{1, t}$ es un shock tecnológico específico al sector $\left(Z_{1, t} \sim \mathcal{N}\left(0, \sigma_{z_{1, t}}^{2}\right)\right.$ ). Dado que el objeto de estudio del problema lo constituyen los ciclos económicos, entonces, se supone que el stock de capital, $K_{1}$, es constante mientras que la cantidad empleada de mano de obra se puede considerar como un factor variable a lo largo del tiempo. La ecuación (6) en términos logarítmicos quedaría de la siguiente manera:

$$
y_{1, t}=z_{1, t}+\theta k_{1}+(1-\theta) l_{1, t}(7)
$$

Si se cree que el objetivo de la empresa exportadora consiste en maximizar las ganancias, entonces, la función de beneficios vendría dada por:

$$
B_{1, t}=E P_{1, t}^{*} Z_{1, t} K_{1}^{\theta} L_{1, t}^{1-\theta}-W_{1, t} L_{1, t}-(r+\delta) K_{1}
$$

2 Excepto cuando se señale lo contrario, las letras minúsculas se utilizarán para denotar los logaritmos naturales de las variables. 
Donde,

$(r+\boldsymbol{\delta})$ es el costo de uso del capital -la tasa de interés real más la tasa de depreciación. Dado que $K_{1}$ es constante, entonces, la condición de primer orden del problema de maximización de (8) viene dado por:

$$
(1-\theta) E P_{1, t}^{*} Z_{1, t} K_{1}^{\theta} L_{1, t}^{-\theta}=W_{1, t}
$$

Tomando logaritmos de esta expresión resulta

$$
\ln (1-\theta)+e+p_{1, t}^{*}+z_{1, t}+\theta k_{1}-\theta l_{1, t}=w_{1, t}
$$

Sustituyendo la ecuación (3) y resolviendo se obtiene:

$$
l_{1, t}=\frac{1}{\theta}\left(-{ }_{i}+p_{1, t}^{*}+\ln (1-\theta)+e(1-v)+\theta k_{1}-v p_{t}^{*}-p_{t}(1-v)+z_{1, t}\right)
$$

Esta ecuación describe la demanda de trabajo en el sector exportador. Sustituyendo este resultado en la ecuación (7), y luego de algunas manipulaciones algebraicas resulta en:

$$
y_{1, t}=-\theta_{1}(1-v) p_{t}-\theta_{1} v p_{t}^{*}+\theta_{1} p_{1, t}^{*}+\theta_{1}(1-v) e+\theta_{1} \quad+\theta_{1} \ln (1-\theta)+\frac{1}{\theta} z_{1, t}+k_{1}(9)
$$

Donde,

$$
\theta_{1}=\frac{1}{\theta}(1-\theta)
$$

Esta ecuación describe (en logaritmos) la función de oferta del sector exportador. Es de destacar la relación positiva entre los logaritmos del precio del producto exportable, $p_{1, t}^{*}$, y su producción y la relación inversa entre los logaritmos del nivel de precios domésticos y el producto exportable.

\subsubsection{Producción en el resto de las actividades}

$\mathrm{Al}$ igual que en la sección precedente, se considera que la producción se lleva a cabo por medio de una tecnología Cobb-Douglas con rendimientos constantes a escala:

$$
\Upsilon_{2, t}=Z_{2, t} K_{2}^{\phi} L_{2, t}^{1-\phi}
$$

Donde,

$0<\theta<1, K_{2}$ y $L_{2, t}$, corresponden a la cantidad de capital y trabajo empleado en el sector en el momento $t \mathrm{y} Z_{2, t}$ es un shock tecnológico específico a la industria petrolera $\left(z_{2, t} \sim \mathcal{N}\left(0, \sigma_{z_{2, t}}^{2}\right)\right)$. Al igual que en el otro sector, el stock de capital, $K_{2}$, es constante mientras que la mano de obra es variable en el corto plazo. Entonces, la ecuación (10) en términos logarítmicos quedaría de la siguiente manera: 


$$
y_{2, t}=z_{2, t}+\phi k_{2}+(1-\phi) l_{2, t}
$$

Bajo el supuesto de maximización de ganancias, entonces, la función de beneficios del sector vendría dada por:

$$
B_{2, t}=P_{t} Z_{2, t} K_{2}^{\phi} L_{2, t}^{1-\phi}-W_{2, t} L_{2, t}-(r+\delta) K_{2}(12)
$$

Dado que $K_{2}$ es constante, entonces, la condición de primer orden del problema de maximización de (12) viene dado por:

$$
(1-\phi) P_{t} Z_{2, t} K_{2}^{\phi} L_{2, t}^{-\phi}=W_{2, t}
$$

Al tomar logaritmos, esta expresión se reduce a

$$
\ln (1-\phi)+p_{t}+z_{2, t}+\phi k_{2}-\phi l_{2, t}=w_{2, t}
$$

Sustituyendo la ecuación (3) y resolviendo, resulta:

$$
l_{2, t}=\frac{1}{\phi}\left(\ln (1-\phi)+p_{t}+z_{2, t}+\phi k_{2}-\left(_{i}+v e+v p_{t}^{*}+(1-v) p_{t}\right)\right)
$$

Esta ecuación describe la demanda de trabajo en el resto de las actividades. Sustituyendo este resultado en la ecuación (11) y después de un poco de álgebra se obtiene:

$$
y_{2, t}=\theta_{2} v p_{t}-\theta_{2} v p_{t}^{*}-\theta_{2} v e+\theta_{2} \ln (1-\phi)-\theta_{2} \quad+\frac{1}{\phi} z_{2, t}+k_{2}
$$

Donde,

$$
\theta_{2}=\frac{1}{\phi}(1-\phi)
$$

$\mathrm{Al}$ igual que en la sección anterior, esta expresión matemática describe (en logaritmos) la función de oferta del sector 2.

\subsubsection{Función de oferta agregada}

La función de oferta agregada se obtiene sustituyendo las ecuaciones (9) y (13) en la ecuación (5):

$$
\begin{gathered}
y_{t}=\left(\theta_{2}-\theta_{1}\left(\frac{1-v}{v}\right)\right) p_{t}-v\left(\theta_{1}+\theta_{2}\right) p_{t}^{*}-\left(\theta_{2}+\theta_{1}\right) \quad-\left(\theta_{2}-\theta_{1}\left(\frac{1-v}{v}\right)\right) e \\
+k_{1}+k_{2}+\theta_{2} \ln (1-\phi)+\theta_{1}(\ln (1-\theta))+\theta_{1} p_{1, t}^{*}+\frac{1}{\theta} z_{1, t}+\frac{1}{\phi} z_{2, t}
\end{gathered}
$$


Esta curva de oferta agregada tendrá una pendiente positiva en el espacio $(y, p)$ siempre que $\theta_{2}-\theta_{1}\left(\frac{1-v}{v}\right)>0$. De igual manera, si la función de oferta tiene pendiente positiva, entonces, una devaluación de la moneda local ocasiona un aumento de la producción del sector exportable, y una disminución del producto en las otras actividades lo que se traduce en un descenso del producto agregado ya que $-\left(\theta_{2}-\theta_{1}\left(\frac{1-v}{v}\right)\right)<0$. Esta ecuación también destaca la baja o nula correlación que existe entre el producto real del sector 1 y el del 2 ante aumentos de los precios del producto de exportación ${ }^{3}$. En otras palabras, un boom en el sector 1 no se traduce necesariamente en una expansión económica en el resto de las actividades.

\subsection{Demanda agregada}

La demanda agregada viene determinada por el equilibrio en el mercado real de bienes y servicios, el equilibrio en el mercado monetario y en el externo, determinado por el saldo de la balanza de pagos. El equilibrio del mercado real viene dado por la expresión (en logs.):

$$
y_{t}=\sigma_{p} p_{1, t}^{*}+\alpha\left(e+p_{t}^{*}-p_{t}\right)-\gamma r_{t}+\sigma_{f} y_{t}^{*}+z_{I S}
$$

Donde:

$y_{t}^{*}$ es el logaritmo del producto real del principal socio comercial ${ }^{4}, \mathrm{r}$ es la tasa de interés real y $z_{I S}$ es un $\operatorname{shock}\left(z_{I S} \sim\left(0, \sigma_{z_{I S}}^{2}\right)\right)$ en cualesquiera de los elementos que conforman el equilibrio del mercado real.

El equilibrio del mercado monetario requiere que la oferta iguale la demanda de saldos reales y viene dado por la siguiente expresión:

$$
m_{t}-p_{t}=\beta_{0} y_{t}-\beta_{1} i_{t}+z_{M}
$$

Donde,

$m_{t}$ es el logaritmo natural de la cantidad de dinero, $\beta_{0}$ y $\beta_{1}$ son parámetros positivos y representan la elasticidad del ingreso real, $y_{t}$, y la semielasticidad de la tasa de interés nominal, $i_{t}, y z_{M}$ es un shock no anticipado en el lado monetario de la economía. La tasa de interés nominal es una tasa de interés ex-ante y se determina de la siguiente manera:

$$
i_{t}=r_{t}+\pi^{e}
$$

3 Véase por ejemplo Cartaya, Sáez y Zavarce (2010) que han encontrado evidencias de la asincronía de los ciclos en la economía venezolana.

4 El principal socio comercial de muchos países latinoamericanos es Estados Unidos. 
Donde,

$\pi^{e}$ es el valor esperado en $t$ de la tasa de inflación en $t+1$. Para simplificar el análisis, supóngase que las expectativas inflacionarias son adaptativas, y por tanto, $\pi^{e}=\pi_{t-1}$. Sustituyendo este parámetro en la expresión anterior, y luego en la ecuación (16), el equilibrio del mercado monetario vendría dado por:

$$
m_{t}-p_{t}=\beta_{0} y_{t}-\beta_{1} r_{t}-\beta_{1} \pi_{t-1}+z_{M}
$$

Seguidamente, con movilidad imperfecta de capitales, el equilibrio externo viene dado por la ecuación de la balanza de pagos. De manera específica:

$$
B_{t}=\sigma_{p} p_{1, t}^{*}+\sigma_{x}\left(e+p_{t}^{*}-p_{t}\right)+\sigma_{f} y_{t}^{*}-\sigma_{y} y_{t}+z_{E X}+\lambda\left(r-r^{*}\right)
$$

Donde,

$\lambda>0$ representa el grado de movilidad del capital y $\sigma_{p}, \sigma_{x}, \sigma_{f}$ y $\sigma_{y}$ son, respectivamente, la elasticidad de la balanza de pagos respecto al precio del petróleo, la elasticidad respecto a cada uno de los componentes de la tasa de cambio real, la elasticidad al producto externo y la de las importaciones al producto doméstico. Al igual que en casos anteriores, $z_{E X} \sim\left(0, \sigma_{z_{E X}^{2}}^{2}\right)$.

\section{Fluctuaciones económicas}

El modelo de oferta y demanda agregada que se presenta se resume en un sistema de cuatro ecuaciones: una para la oferta agregada (14), y tres para la demanda agregada (15), (17) y (18). Bajo el supuesto de una paridad cambiaria fija, entonces, las variables endógenas son: $y_{t}, m_{t}, r_{t}$ y $p_{t}$. En la presente investigación se usará el álgebra matricial para calcular los impactos. Para ello se procede, en primer lugar, a calcular la diferencial total del sistema y a separar las variables endógenas de las exógenas; segundo, se escribe el sistema resultante en forma matricial; y finalmente, se calculan los impactos.

El sistema totalmente diferenciado quedaría de la siguiente manera:

$$
\begin{gathered}
d y_{t}-\left(\theta_{2}-\theta_{1}\left(\frac{1-v}{v}\right)\right) d p_{t}=-v\left(\theta_{1}+\theta_{2}\right) d p_{t}^{*}-\left(\theta_{2}-\theta_{1}\left(\frac{1-v}{v}\right)\right) d e \\
+\theta_{1} d p_{1, t}^{*}+\frac{1}{\theta} d z_{1, t}+\frac{1}{\phi} d z_{2, t} \\
d y_{t}+\alpha d p_{t}+\gamma d r_{t}=\sigma_{p} d p_{1, t}^{*}+\alpha\left(d e+d p_{t}^{*}\right)+\sigma_{f} d y_{t}^{*}+d z_{I S}
\end{gathered}
$$


ISSN: 0124-3551 / Año 15, No 22 / enero-junio / pp. 141-160

$$
\begin{gathered}
\beta_{1} d r_{t}-\beta_{0} d y_{t}+d m_{t}-d p_{t}=d z_{M}(21) \\
-\sigma_{x} d p_{t}-\sigma_{y} d y_{t}+\lambda d r_{t}=-\sigma_{p} d p_{1, t}^{*}-\sigma_{x}\left(d e+d p_{t}^{*}\right)-\sigma_{f} d y_{t}^{*}-d z_{E X}+\lambda d r^{*}
\end{gathered}
$$

Seguidamente, el sistema en forma matricial:

$$
\begin{gathered}
{\left[\begin{array}{cccc}
1 & -\left(\theta_{2}-\theta_{1}\left(\frac{1-v}{v}\right)\right) & 0 & 0 \\
1 & \alpha & 0 & \gamma \\
-\beta_{0} & -1 & 1 & \beta_{1} \\
-\sigma_{y} & -\sigma_{x} & 0 & \lambda
\end{array}\right]\left[\begin{array}{c}
d y_{t} \\
d p_{t} \\
d m_{t} \\
d r_{t}
\end{array}\right]=} \\
{\left[\begin{array}{c}
-v\left(\theta_{1}+\theta_{2}\right) d p_{t}^{*}-\left(\theta_{2}-\theta_{1}\left(\frac{1-v}{v}\right)\right) d e+\theta_{1} d p_{1, t}^{*}+\frac{1}{\theta} d z_{1, t}+\frac{1}{\phi} d z_{2, t} \\
\sigma_{p} d p_{1, t}^{*}+\alpha\left(d e+d p_{t}^{*}\right)+\sigma_{f} d y_{t}^{*}+d z_{I S} \\
d z_{M} \\
-\sigma_{p} d p_{1, t}^{*}-\sigma_{x}\left(d e+d p_{t}^{*}\right)-\sigma_{f} d y_{t}^{*}-d z_{E X}+\lambda d r^{*}
\end{array}\right]}
\end{gathered}
$$

Definase

$$
D=\left[\begin{array}{cccc}
1 & -\left(\theta_{2}-\theta_{1}\left(\frac{1-v}{v}\right)\right) & 0 & 0 \\
1 & \alpha & 0 & \gamma \\
-\beta_{0} & -1 & 1 & \beta_{1} \\
-\sigma_{y} & -\sigma_{x} & 0 & \lambda
\end{array}\right]
$$

Entonces, el determinante de D sería igual a:

$$
|D|=\alpha \lambda+\gamma \sigma_{x}+\left(\theta_{2}-\theta_{1}\left(\frac{1-v}{v}\right)\right)\left(\lambda+\gamma \sigma_{y}\right)>0
$$

Este resultado es muy importante y se utilizará más adelante en la obtención y evaluación de los impactos.

\subsection{Shock doméstico real}

Para determinar el impacto de un shock doméstico en el sector real (un aumento del gasto, una disminución de impuestos, un cambio favorable en el consumo hacia los bienes producidos domésticamente, un aumento de la inversión, etc.), se supone $d z_{I S}>0$ y el resto de las diferenciales de las variables exógenas toman el valor de cero; es decir:

$$
d p_{t}^{*}=d e=d p_{1, t}^{*}=d z_{1, t}=d z_{2, t}=d y_{t}^{*}=d z_{M}=d z_{E X}=0 .
$$


De esta manera, el sistema se reduce a:

$$
\left[\begin{array}{cccc}
1 & -\left(\theta_{2}-\theta_{1}\left(\frac{1-v}{v}\right)\right) & 0 & 0 \\
1 & \alpha & 0 & \gamma \\
-\beta_{0} & -1 & 1 & \beta_{1} \\
-\sigma_{y} & -\sigma_{x} & 0 & \lambda
\end{array}\right]\left[\begin{array}{c}
d y_{t} \\
d p_{t} \\
d m_{t} \\
d r_{t}
\end{array}\right]=\left[\begin{array}{c}
0 \\
d z_{I S} \\
0 \\
0
\end{array}\right]
$$

Dividiendo por $d z_{I S}$

$$
\left[\begin{array}{cccc}
1 & -\left(\theta_{2}-\theta_{1}\left(\frac{1-v}{v}\right)\right) & 0 & 0 \\
1 & \alpha & 0 & \gamma \\
-\beta_{0} & -1 & 1 & \beta_{1} \\
-\sigma_{y} & -\sigma_{x} & 0 & \lambda
\end{array}\right]\left[\begin{array}{c}
d y_{t} / d z_{I S} \\
d p_{t} / d z_{I S} \\
d m_{t} / d z_{I S} \\
d r_{t} / d z_{I S}
\end{array}\right]=\left[\begin{array}{c}
0 \\
1 \\
0 \\
0
\end{array}\right]
$$

Donde, $d y_{t} / d z_{I S}, d p_{t} / d z_{I S}, d m_{t} / d z_{I S} \mathrm{y} d r_{t} / d z_{I S}$ son los impactos sobre el producto real, el nivel de precios, la oferta monetaria y la tasa de interés real, respectivamente. Dado que el sistema es lineal, su solución se puede hallar por cualquier método. En el presente trabajo se utilizará el método de Cramer para hallar la solución de las incógnitas del modelo. Los resultados se muestran a continuación:

$$
\begin{gathered}
\frac{d y_{t}}{d z_{I S}}=\frac{\left(\theta_{2}-\theta_{1}\left(\frac{1-v}{v}\right)\right) \lambda}{|D|}>0 \\
\frac{d p_{t}}{d z_{I S}}=\frac{\lambda}{|D|}>0 \\
\frac{d m_{t}}{d z_{I S}}=\frac{\left(\theta_{2}-\theta_{1}\left(\frac{1-v}{v}\right)\right)\left(\beta_{0} \lambda-\beta_{1} \sigma_{y}\right)+\lambda-\beta_{1} \sigma_{x}}{|D|} \gtreqless 0
\end{gathered}
$$

Este resultado dependerá del signo de $\left(\beta_{0} \lambda-\beta_{1} \sigma_{y}\right)$ y de $\lambda-\beta_{1} \sigma_{x}$. Si estos valores son positivos, es decir, si $\frac{\beta_{0}}{\beta_{1}}>\frac{\sigma_{y}}{\lambda}$, entonces, la economía se enfrenta a una relativa elevada movilidad de capitales y la oferta monetaria aumentará ante el efecto de la política. Si por el contrario, los valores son negativos, entonces, la oferta monetaria disminuirá con una expansión fiscal. Finalmente,

$$
\frac{d r_{t}}{d z_{I S}}=\frac{1}{v|D|}\left(v \sigma_{x}-\theta_{1} \sigma_{y}+v \theta_{1} \sigma_{y}+v \theta_{2} \sigma_{y}\right) \gtreqless 0
$$

El efecto sobre la tasa de interés será positivo, si $v \sigma_{x}+v \theta_{2} \sigma_{y}>\theta_{1} \sigma_{y}(v-1)$. 
Es decir, un shock doméstico real produciría un aumento del producto real y del nivel de precios. El costo de una expansión económica por medio de un aumento del gasto se refleja en un incremento del nivel de precios en la economía, mientras que los efectos sobre la tasa de interés y le oferta de dinero dependerán del grado de movilidad de capitales. Estos son resultados estándar en la literatura económica.

\subsection{Shocks monetarios}

Bajo un régimen de cambio fijo, una política monetaria expansiva es completamente ineficaz para producir aumentos en el producto real y el empleo, ya que el crecimiento de la cantidad de dinero produce una presión al alza de la tasa de cambio y el Banco Central, para mantener la tasa de cambio fija, debe entonces vender divisas, reduciendo con ello, la cantidad de dinero en circulación. Como consecuencia de la ineficacia de la política monetaria, los únicos shocks monetarios a considerar están relacionados con la política cambiaria; específicamente, se evalúa el impacto de una devaluación de la moneda local $(d e>0)$, ceteris paribus, $\left(d p_{t}^{*}=d z_{M}=d p_{1, t}^{*}=d z_{1, t}=d z_{2, t}=d y_{t}^{*}=d z_{I S}=d z_{E X}=0\right)$. En otras palabras, el sistema formado por las ecuaciones (19), (20), (21) y (22) queda de la siguiente manera:

$$
\left[\begin{array}{cccc}
1 & -\left(\theta_{2}-\theta_{1}\left(\frac{1-v}{v}\right)\right) & 0 & 0 \\
1 & \alpha & 0 & \gamma \\
-\beta_{0} & -1 & 1 & \beta_{1} \\
-\sigma_{y} & -\sigma_{x} & 0 & \lambda
\end{array}\right]\left[\begin{array}{c}
d y_{t} \\
d p_{t} \\
d m_{t} \\
d r_{t}
\end{array}\right]=\left[\begin{array}{c}
-\left(\theta_{2}-\theta_{1}\left(\frac{1-v}{v}\right)\right) d e \\
\alpha d e \\
0 \\
-\sigma_{x} d e
\end{array}\right]
$$

que se reduce a:

$$
\left[\begin{array}{cccc}
1 & -\left(\theta_{2}-\theta_{1}\left(\frac{1-v}{v}\right)\right) & 0 & 0 \\
1 & \alpha & 0 & \gamma \\
-\beta_{0} & -1 & 1 & \beta_{1} \\
-\sigma_{y} & -\sigma_{x} & 0 & \lambda
\end{array}\right]\left[\begin{array}{c}
d y_{t} / d e \\
d p_{t} / d e \\
d m_{t} / d e \\
d r_{t} / d e
\end{array}\right]=\left[\begin{array}{c}
-\left(\theta_{2}-\theta_{1}\left(\frac{1-v}{v}\right)\right) \\
\alpha \\
0 \\
-\sigma_{x}
\end{array}\right]
$$

Por tanto, los impactos sobre las variables endógenas vendrían dados por:

$$
\begin{gathered}
\frac{d y_{t}}{d e}=0 \\
\frac{d p_{t}}{d e}=\frac{\left(\theta_{2}-\theta_{1}\left(\frac{1-v}{v}\right)\right)\left(\gamma \sigma_{y}+\lambda\right)+\gamma \sigma_{x}+\lambda \alpha}{|D|}>0
\end{gathered}
$$




$$
\begin{gathered}
\frac{d m_{t}}{d e}=\frac{\left(\theta_{2}-\theta_{1}\left(\frac{1-v}{v}\right)\right)\left(\gamma \sigma_{y}+\lambda\right)+\gamma \sigma_{x}+\lambda \alpha}{|D|}>0 \\
\frac{d r_{t}}{d e}=0
\end{gathered}
$$

De acuerdo con estos resultados, los efectos de una devaluación en una economía pequeña monoexportadora sólo ocasionan un aumento de la oferta monetaria e inflación en el corto plazo. Este resultado es contrario al encontrado, por ejemplo, en modelos de economías con un sólo sector productivo exportador o al observado en el mundo real en economías más diversificadas y desarrolladas. Este resultado también pudiera convertirse en una de las razones por las que algunos sectores productivos en algunos países latinoamericanos se oponen políticamente a una devaluación de sus monedas. Por otra parte, aunque no se aprecia en el modelo, la devaluación de la moneda local puede ser utilizada para corregir desequilibrios de la balanza de pagos o para controlar la presión sobre el tipo de cambio y las reservas internacionales.

\subsection{Shocks externos}

\section{Expansión económica externa}

En muchos países de Latinoamérica el principal socio comercial es Estados Unidos. Entonces, una expansión económica en el resto del mundo puede ser interpretada como un crecimiento económico de su principal socio comercial. En términos del modelo esto implicaría que $d y_{t}^{*}>0$, céteris paribus, lo cual implica que: $d p_{t}^{*}=d z_{M}=d p_{1, t}^{*}=d z_{1, t}=d z_{2, t}=d e=d z_{I S}=d z_{E X}=0$

$$
\left[\begin{array}{cccc}
1 & -\left(\theta_{2}-\theta_{1}\left(\frac{1-v}{v}\right)\right) & 0 & 0 \\
1 & \alpha & 0 & \gamma \\
-\beta_{0} & -1 & 1 & \beta_{1} \\
-\sigma_{y} & -\sigma_{x} & 0 & \lambda
\end{array}\right]\left[\begin{array}{c}
d y_{t} \\
d p_{t} \\
d m_{t} \\
d r_{t}
\end{array}\right]=\left[\begin{array}{c}
0 \\
\sigma_{f} d y_{t}^{*} \\
0 \\
-\sigma_{f} d y_{t}^{*}
\end{array}\right]
$$

al dividir las filas de la matriz por $d y_{t}^{*}$ se obtiene:

$$
\left[\begin{array}{cccc}
1 & -\left(\theta_{2}-\theta_{1}\left(\frac{1-v}{v}\right)\right) & 0 & 0 \\
1 & \alpha & 0 & \gamma \\
-\beta_{0} & -1 & 1 & \beta_{1} \\
-\sigma_{y} & -\sigma_{x} & 0 & \lambda
\end{array}\right]\left[\begin{array}{c}
d y_{t} / d y_{t}^{*} \\
d p_{t} / d y_{t}^{*} \\
d m_{t} / d y_{t}^{*} \\
d r_{t} / d y_{t}^{*}
\end{array}\right]=\left[\begin{array}{c}
0 \\
\sigma_{f} \\
0 \\
-\sigma_{f}
\end{array}\right]
$$


Despejando las incógnitas resulta:

$$
\begin{gathered}
\frac{d y_{t}}{d y_{t}^{*}}=\frac{\sigma_{f}\left(\theta_{2}-\theta_{1}\left(\frac{1-v}{v}\right)\right)(\gamma+\lambda)}{|D|}>0 \\
\frac{d p_{t}}{d y_{t}^{*}}=\frac{\sigma_{f}(\lambda+\gamma)}{|D|}>0 \\
\frac{d m_{t}}{d y_{t}^{*}}=\frac{\sigma_{f}\left(\theta_{2}-\theta_{1}\left(\frac{1-v}{v}\right)\right)\left(\beta_{1}\left(\sigma_{y}-\sigma_{f}\right)-\beta_{0}(\lambda+\gamma)\right)-\sigma_{f}\left(\lambda+\gamma-\beta_{1}\left(\sigma_{x}+\alpha\right)\right)}{|D|} \gtreqless 0 \\
\frac{d r_{t}}{d y_{t}^{*}}=\frac{\sigma_{f}\left(\left(\theta_{2}-\theta_{1}\left(\frac{1-v}{v}\right)\right)\left(\sigma_{y}-1\right)+\left(\alpha+\sigma_{x}\right)\right)}{|D|} \gtreqless 0
\end{gathered}
$$

Estos resultados son consistentes con los hallazgos de Mora (2008; 2002) e indican que una expansión económica en Estados Unidos produciría un incremento de la actividad económica interna con un crecimiento del nivel de precios, lo cual implica que los ciclos económicos de Estados Unidos y de la economía están correlacionados. Desde el punto de vista de este modelo, la correlación entre los movimientos del producto real y del nivel de precios con sus respectivas contrapartes de Estados Unidos dependerán evidentemente del tamaño de la elasticidad del producto externo, $\sigma_{f}$. Si $\sigma_{f} \rightarrow 0$, entonces, los efectos de la expansión económica en Estados Unidos serán pequeños o imperceptibles.

\section{Aumento de precio del bien exportable}

Dada la importancia del producto de exportación en la economía se estudian ahora los efectos de un aumento de su precio; es decir, $d p_{1, t}^{*}>0$, céteris páribus, por lo que:

$$
\begin{gathered}
d p_{t}^{*}=d z_{M}=d y_{t}^{*}=d z_{1, t}=d z_{2, t}=d e=d z_{I S}=d z_{E X}=0 \\
{\left[\begin{array}{cccc}
1 & -\left(\theta_{2}-\theta_{1}\left(\frac{1-v}{v}\right)\right) & 0 & 0 \\
1 & \alpha & 0 & \gamma \\
-\beta_{0} & -1 & 1 & \beta_{1} \\
-\sigma_{y} & -\sigma_{x} & 0 & \lambda
\end{array}\right]\left[\begin{array}{c}
d y_{t} \\
d p_{t} \\
d m_{t} \\
d r_{t}
\end{array}\right]=\left[\begin{array}{c}
\theta_{1} d p_{1, t}^{*} \\
\sigma_{p} d p_{1, t}^{*} \\
0 \\
-\sigma_{p} d p_{1, t}^{*}
\end{array}\right]}
\end{gathered}
$$

y que se reduce a: 


$$
\left[\begin{array}{cccc}
1 & -\left(\theta_{2}-\theta_{1}\left(\frac{1-v}{v}\right)\right) & 0 & 0 \\
1 & \alpha & 0 & \gamma \\
-\beta_{0} & -1 & 1 & \beta_{1} \\
-\sigma_{y} & -\sigma_{x} & 0 & \lambda
\end{array}\right]\left[\begin{array}{c}
d y_{t} / d p_{1, t}^{*} \\
d p_{t} / d p_{1, t}^{*} \\
d m_{t} / d p_{1, t}^{*} \\
d r_{t} / d p_{1, t}^{*}
\end{array}\right]=\left[\begin{array}{c}
\theta_{1} \\
\sigma_{p} \\
0 \\
-\sigma_{p}
\end{array}\right]
$$

que al despejar las variables desconocidas $d y_{t} / d p_{1, t}^{*}, d p_{t} / d p_{1, t}^{*}, d m_{t} / d p_{1, t}^{*}$ y $d r_{t} / d p_{1, t}^{*}$ se obtiene:

$$
\begin{aligned}
& \frac{d y_{t}}{d p_{1, t}^{*}}=\frac{\left(\theta_{2}-\theta_{1}\left(\frac{1-v}{v}\right)\right) \sigma_{p}(\gamma+\lambda)+\theta_{1}\left(\alpha \lambda+\sigma_{x} \gamma\right)}{|D|}>0 \\
& \frac{d p_{t}}{d p_{1, t}^{*}}=\frac{\left(\sigma_{p} \frac{\beta_{1}+\gamma m_{m}}{\beta_{1}}\right)-\theta_{1}\left(\frac{\beta_{1}+\gamma \beta_{0}+\gamma \sigma_{y} m_{m}}{\beta_{1}}\right)}{|D|} \gtreqless 0 \\
& \frac{d m_{t}}{d p_{1, t}^{*}}=\frac{\left(\theta_{2}-\theta_{1}\left(\frac{1-v}{v}\right)\right)\left(\sigma_{p} \beta_{0}(\lambda+\gamma)+\sigma_{p} \beta_{1}\left(1-\sigma_{y}\right)\right)+\theta_{1} \beta_{1}\left(\sigma_{x}-\alpha \sigma_{y}\right)+\theta_{1} \beta_{0}\left(\alpha \lambda+\gamma \sigma_{x}\right)}{|D|} \\
& +\frac{\sigma_{p}(\gamma+\lambda)-\theta_{1}\left(\gamma \sigma_{y}+\lambda\right)+\beta_{1} \sigma_{p}\left(\alpha-\sigma_{x}\right)}{|D|} \gtreqless 0 \\
& \frac{d r_{t}}{d p_{1, t}^{*}}=\frac{\theta_{1}\left(\alpha \sigma_{y}-\sigma_{x}\right)+\sigma_{p}\left(\sigma_{x}-\alpha-\left(\theta_{2}-\theta_{1}\left(\frac{1-v}{v}\right)\right)\left(1-\sigma_{y}\right)\right)}{|D|} \gtreqless 0
\end{aligned}
$$

De acuerdo con estos resultados, el aumento del precio del producto de exportación tiene efectos positivos sobre la actividad económica ya que no solo aumenta la oferta agregada, sino también la demanda agregada. Esto se traduciría en un crecimiento del producto real; no obstante, los efectos sobre el nivel de precios, la oferta monetaria y la tasa de interés son ambiguos y dependerán del grado de movilidad de capitales, de las elasticidades con respecto a las variables externas y la elasticidad del tipo de cambio real.

\subsection{Shocks domésticos de productividad}

Desde el punto de vista de la oferta agregada, sólo se consideran shocks de productividad en la actividad exportadora, y en el sector de otras actividades económicas. 


\section{En el sector exportador}

Supóngase $d z_{1, t}>0 \quad$ y ninguna alteración en el resto de variables exógenas $\left(d p_{t}^{*}=d p_{1, t}^{*}=d z_{M}=d y_{t}^{*}=d z_{2, t}=d e=d z_{I S}=d z_{E X}=0\right)$.

$$
\left[\begin{array}{cccc}
1 & -\left(\theta_{2}-\theta_{1}\left(\frac{1-v}{v}\right)\right) & 0 & 0 \\
1 & \alpha & 0 & \gamma \\
-\beta_{0} & -1 & 1 & \beta_{1} \\
-\sigma_{y} & -\sigma_{x} & 0 & \lambda
\end{array}\right]\left[\begin{array}{c}
d y_{t} \\
d p_{t} \\
d m_{t} \\
d r_{t}
\end{array}\right]=\left[\begin{array}{c}
\frac{1}{\theta} d z_{1, t} \\
0 \\
0 \\
0
\end{array}\right]
$$

dividiendo por $d z_{1, t}$ resulta:

$$
\left[\begin{array}{cccc}
1 & -\left(\theta_{2}-\theta_{1}\left(\frac{1-v}{v}\right)\right) & 0 & 0 \\
1 & \alpha & 0 & \gamma \\
-\beta_{0} & -1 & 1 & \beta_{1} \\
-\sigma_{y} & -\sigma_{x} & 0 & \lambda
\end{array}\right]\left[\begin{array}{c}
d y_{t} / d z_{1, t} \\
d p_{t} / d z_{1, t} \\
d m_{t} / d z_{1, t} \\
d r_{t} / d z_{1, t}
\end{array}\right]=\left[\begin{array}{c}
\frac{1}{\theta} \\
0 \\
0 \\
0
\end{array}\right]
$$

Entonces, los cambios en las variables endógenas vendrían dados por:

$$
\begin{aligned}
& \frac{d y_{t}}{d z_{1, t}}=\frac{\left(\alpha \lambda+\sigma_{x} \gamma\right) \frac{1}{\theta}}{|D|}>0 \\
& \frac{d p_{t}}{d z_{1, t}}=\frac{-\left(\frac{1}{\theta}\right)\left(\gamma \sigma_{y}+\lambda\right)}{|D|}<0 \\
& \frac{d m_{t}}{d z_{1, t}}=\frac{\frac{1}{\theta}\left(\beta_{0}\left(\alpha \lambda+\sigma_{x} \gamma\right)+\beta_{1}\left(\sigma_{x}-\alpha \sigma_{y}\right)-\left(\lambda+\sigma_{y} \gamma\right)\right)}{|D|} \gtreqless 0 \\
& \frac{d r_{t}}{d z_{1, t}}=\frac{\left(\frac{1}{\theta}\right)\left(\alpha \sigma_{y}-\sigma_{x}\right)}{|D|} \gtreqless 0
\end{aligned}
$$

Como se observa en estos resultados, el aumento de la productividad en el sector exportador trae como consecuencia un crecimiento del producto real y una disminución del nivel de precios en la economía doméstica; no obstante, los efectos sobre la oferta monetaria y la tasa de interés se definen en función de las elasticidades a las variables externas y el grado de movilidad de capitales. 


\section{En el sector de otras actividades económicas}

Supóngase $d z_{2, t}>0$ y ninguna alteración en el resto de variables exógenas $\left(d p_{t}^{*}=d p_{1, t}^{*}=d z_{M}=d y_{t}^{*}=d z_{1, t}=d e=d z_{I S}=d z_{E X}=0\right)$.

$$
\left[\begin{array}{cccc}
1 & -\left(\theta_{2}-\theta_{1}\left(\frac{1-v}{v}\right)\right) & 0 & 0 \\
1 & \alpha & 0 & \gamma \\
-\beta_{0} & -1 & 1 & \beta_{1} \\
-\sigma_{y} & -\sigma_{x} & 0 & \lambda
\end{array}\right]\left[\begin{array}{c}
d y_{t} / d z_{2, t} \\
d p_{t} / d z_{2, t} \\
d m_{t} / d z_{2, t} \\
d r_{t} / d z_{2, t}
\end{array}\right]=\left[\begin{array}{c}
\frac{1}{\phi} \\
0 \\
0 \\
0
\end{array}\right]
$$

y los efectos sobre el productor real, el nivel de precios, la oferta monetaria y la tasa de interés vendrían dados por:

$$
\begin{gathered}
\frac{d y_{t}}{d z_{2, t}}=\frac{\left(\alpha \lambda+\sigma_{x} \gamma\right) \frac{1}{\phi}}{|D|}>0 \\
\frac{d p_{t}}{d z_{2, t}}=\frac{-\left(\frac{1}{\phi}\right)\left(\gamma \sigma_{y}+\lambda\right)}{|D|}<0 \\
\frac{d m_{t}}{d z_{2, t}}=\frac{\frac{1}{\phi}\left(\beta_{0}\left(\alpha \lambda+\sigma_{x} \gamma\right)+\beta_{1}\left(\sigma_{x}-\alpha \sigma_{y}\right)-\left(\lambda+\sigma_{y} \gamma\right)\right)}{|D|} \gtreqless 0 \\
\frac{d r_{t}}{d z_{2, t}}=\frac{\left(\frac{1}{\phi}\right)\left(\alpha \sigma_{y}-\sigma_{x}\right)}{|D|} \gtreqless 0
\end{gathered}
$$

$\mathrm{Al}$ igual que en el caso anterior, el aumento de la productividad en el sector de otras actividades económicas trae como consecuencia un incremento del producto real y una disminución del nivel de precios en la economía doméstica. Cualitativamente estos dos shocks tienen impactos similares, no obstante, desde el punto de vista cuantitativo la magnitud es variable dada la importancia relativa que tiene la actividad exportadora en la economía.

Finalmente, es necesario destacar que los últimos dos shocks considerados se comportan como shocks puros de oferta ya que en ambos casos la demanda agregada no cambia. 


\section{Implicaciones de política económica}

Dados los resultados obtenidos en la sección anterior, el uso de una política fiscal expansiva tiene consecuencias positivas sobre el producto real, pero a expensas de mayores precios en la economía mientras que una devaluación dejaría la actividad económica real igual, pero aumentaría la tasa de inflación. Contrario a lo que ocurre en muchos países, una devaluación es una medida correctiva de los desequilibrios macroeconómicos internos y externos muy útil, pero en este caso particular, los efectos sobre el producto real son nulos o insignificantes, al menos en el corto plazo. Tal vez, la devaluación corrija los desequilibrios externos, pero evidentemente los efectos sobre la economía interna no favorecen la actividad productiva. Adicionalmente, este resultado también revela una de las razones por las que los empresarios y consumidores de algunos países de América Latina nunca han visto con buenos ojos la devaluación de la moneda local, ya que esta aumenta no solo los costos de producción, sino también se transmite al nivel de precios por el aumento endógeno de la oferta monetaria.

Por otra parte, la expansión económica en el resto del mundo, principalmente en Estados Unidos, tiene efectos positivos sobre la economía doméstica aunque los precios también tienden a aumentar. Desde el punto de vista real, los resultados apuntan hacia una correlación positiva entre los ciclos económicos de ambas economías al menos cualitativamente, pero tal vez, desde el punto de vista cuantitativo esta correlación no sea estadísticamente significativa. Lo que sí es indiscutible es que, por ser esta una economía pequeña, la expansión de la actividad económica doméstica no afecta la economía de Estados Unidos.

Los aumentos de los precios del producto de exportación favorecen la actividad económica, pero sus efectos sobre el nivel de precios es ambiguo. La evidencia empírica en economías pequeñas, como por ejemplo la venezolana, señala que los aumentos de precios del petróleo inciden de manera positiva en el nivel general de precios.

Respecto a los shocks de productividad, estos son shocks puros. Es decir, cuando ocurren estos cambios en la productividad de cualquiera de los sectores, la oferta agregada se ve afectada, pero estos cambios no afectan la demanda agregada. Entonces, la actividad económica real se ve favorecida y los precios tienden a contraerse.

\section{Conclusiones}

Este trabajo presenta un modelo teórico de oferta y demanda agregada para una economía pequeña suponiendo que esta está formada por dos sectores productivos (exportador y otras actividades), con un régimen de cambio fijo y movilidad imperfecta de capitales.El propósito principal del modelo consiste en determinar los impactos que distintos shocks (domésticos y externos) pudieran tener sobre los precios y la actividad 
económica real. Los resultados en su mayoría son consistentes con los postulados por la teoría económica. No obstante, existen dos resultados muy importantes que vale la pena mencionar: los efectos de una devaluación y un aumento de los precios del producto de exportación. En primer lugar, una devaluación de la moneda local no tiene ningún efecto sobre el producto real y aumenta el nivel de precios, al menos en el corto plazo. Este probablemente es el resultado más importante de este trabajo, ya que es consistente con los hechos y con la evidencia empírica citada en la literatura económica para el caso de algunos países latinoamericanos. Segundo, el aumento de los precios del producto de exportación muestra dos efectos simultáneos. Afecta la oferta agregada por el estímulo a la producción del sector, y por otro lado, incrementa la demanda agregada. Dependiendo de cuál efecto domine, entonces, los precios domésticos pudieran aumentar, disminuir o permanecer inalterados.

\section{Referencias}

Blanchard, O. J. y Watson, M. W. (1986). “Are business cycles all alike?” En Gordon, R. J. (ed.) (1986), pp. 123-179.

Blanchard, O. J. y Quah, D. (1989). "The dynamic effects of aggregate demand and supply disturbances." American Economic Review, 79, pp. 655-673.

Blanchard, O. J. (1989). "A traditional interpretation of macroeconomic fluctuations." American Economic Review, 79, pp. 1146-1164.

Cartaya, V., Sáez, F. y Zavarce, H. (2010). "Ciclos de actividad económica y comovimientos sectoriales en Venezuela." Serie documentos de trabajo, 110. Caracas: Banco Central de Venezuela.

Cecchetti, S. G. y Karras, G. (1992). "Sources of output fluctuations during the interwar period: Further evidence on the causes of the great depression." National Bureau of Economic Research, Working Paper 4049.

Cooley, T. F., y Hansen, G. D. (1997). "Unanticipated money growth and the business cycle reconsidered." Journal of Money, Credit and Banking, 29, 4 (part 2: Dynamic effects of Monetary Policy (November)), pp. 624-648.

Fleming, J. M. (1962). "Domestic financial policy under fixed and under floating exchange rates". IMF Staff Papers, 9, pp. 369-379.

Gali, J. (1999). "Technology, employment, and the business cycle: Do technology shocks explain aggregate fluctuations?" American Economic Review, 89 (March), pp. 249-271. Gordon, R. J. (ed). (1986). The American business cycle: Continuity and change. Chicago. The University of Chicago Press. 
ISSN: 0124-3551 / Año 15, No 22 / enero-junio / pp. 141-160

Karras, G.(1993). "Sources of U.S. macroeconomic fluctuations: 1973-1989." Journal of Macroeconomics, 15, pp. 47-68.

Karras, G. (1994). "Sources of business cycles in Europe: 1960-1988. Evidence from France, Germany, and the United Kingdom." European Economic Reviere, 38, pp. 1763-1778.

King, R. G., Plosser, C. I., Stock, J. H., y Watson, M. W. (1991). "Stochastic trends and economic fluctuations." American Economic Reviere, 81, 4 (September), pp. 819-840.

Lorenzoni, G. (2009). "A theory of demand shocks." American Economic Reviere, 99, 5 (December), pp. 2050-2084.

Mora, J. U. (2008). "Relative importance of foreign and domestic shocks in the Venezuelan economy." Economía, XXXIII, 25 (enero-junio, 2008), pp. 61-86

Mora, J. U. (2002). Inflation, exchange rate instability and balance of payment deficits in Venezuela: A VAR approach. Ph. D. Dissertation. Chicago: University of Illinois at Chicago.

Mundell, R. A. (1963). "Capital mobility and stabilization policy under fixed and flexible exchange rates". Canadian Fournal of Economics and Political Science”, 29, pp. 475-485.

Pagliacci, C. y Barráez, D. (2009). "Oferta y demanda agregada: Una representación de espacio de estados y cambios de régimen. Serie documentos de trabajo, 105. Caracas: Banco Central de Venezuela.

Pagliacci, C. y Ruda, M. (2004). “'Tienen efectos las acciones de política monetaria? Un análisis de intencionalidad." Serie documentos de trabajo, 67. Caracas: Banco Central de Venezuela.

Saez F. J. y Puch, L. A. (2004). "Shocks externos y fluctuaciones en una economía petrolera. Serie documentos de trabajo, 59. Caracas: Banco Central de Venezuela. 